\title{
Crowd motion paradigm modeled by a bilevel sweeping control problem
}

\author{
Tan H. Cao ${ }^{1}$, Nathalie T. Khalil ${ }^{2}$, Boris S. Mordukhovich ${ }^{3}$, Dao Nguyen $^{3}$, and Fernando Lobo Pereira ${ }^{2}$
}

\begin{abstract}
This article concerns an optimal crowd motion control problem in which the crowd features a structure given by its organization into $N$ groups (participants) each one spatially confined in a set. The overall optimal control problem consists in driving the ensemble of sets as close as possible to a given point (the "exit") while the population in each set minimizes its control effort subject to its sweeping dynamics with a controlled state dependent velocity drift. In order to capture the conflict between the goal of the overall population and those of the various groups, the problem is cast as a bilevel optimization framework. A key challenge of this problem consists in bringing together two quite different paradigms: bilevel programming and sweeping dynamics with a controlled drift. Necessary conditions of optimality in the form of a Maximum Principle of Pontryagin in the Gamkrelidze framework are derived. These conditions are then used to solve a simple illustrative example with two participants, emphasizing the interaction between them.
\end{abstract}

\section{INTRODUCTION}

Problems modeled as a bilevel optimization, with dynamics featuring a sweeping process control arise naturally in numerous applications. For instance in managing the motion of structured crowds organized in groups, in operating teams of drones providing complementary services in a shared confined space, in nanoferro-electric technologies for functional improvement of mobile electronic devices, among many others. In all those examples, the problem can be represented as a bilevel optimization, with dynamics modeling some structure formed by a set of groups with distinct properties and confined to controlled bounded moving subsets, giving rise to a sweeping process control phenomena.

The purpose of this article is to present a bilevel sweeping control problem through a model arising in the management of structured crowd motions on the plane. This framework, bringing together bilevel optimization and controlled sweeping processes, was addressed for the first time in [1]. By structured crowd we refer to a population organized into group of sets. As an example, let us imagine a population trying to exit a certain space with the shortest possible path. This population is organized into groups of sets, each moving along a trajectory $y_{i}$ prescribed by a coordinator in order to reach the exit. The population in each group has to remain

\footnotetext{
${ }^{1}$ T.H. Cao is with Department of Applied Mathematics and Statistics, SUNY (State University of New York) Korea tan.cao@stonybrook. edu

${ }^{2}$ N.T. Khalil and F.L. Pereira are with SYSTEC, Faculty of Electrical Engineering, Porto University, and with the Institute for Systems and Robotics, 4200-465 Porto, Portugal khalil.t.nathalie@gmail.com, flp@fe.up.pt

${ }^{3}$ B.S. Mordukhovich and D. Nguyen are with Department of Mathematics, Wayne State University, USA boris@math.wayne.edu, dao. nguyen 2 @wayne. edu
}

inside its own moving set, while minimizing its effort to achieve this. In this context, we formulate a bilevel problem coupled with a sweeping control process: the upper level defines the direction of each group (or set) with the goal of driving the ensemble of sets as close as possible to the exit, while avoiding any overlapping between them, and the lower level problem where each group population has to stay confined to its moving group, via a motion modeled by a sweeping control process, while minimizing its control effort to achieve this.

To simplify, we take the groups to be $N$ disks, in the plane, of the same radius $R$. Each disk is subject to a translation vector $y^{i} \in \mathbb{R}^{2}$ representing its linear motion direction. The population in each disk will be presented by its "representative" position, $x^{i} \in \mathbb{R}^{2}$. The exit set the origin. The problem is illustrated in Figure 1

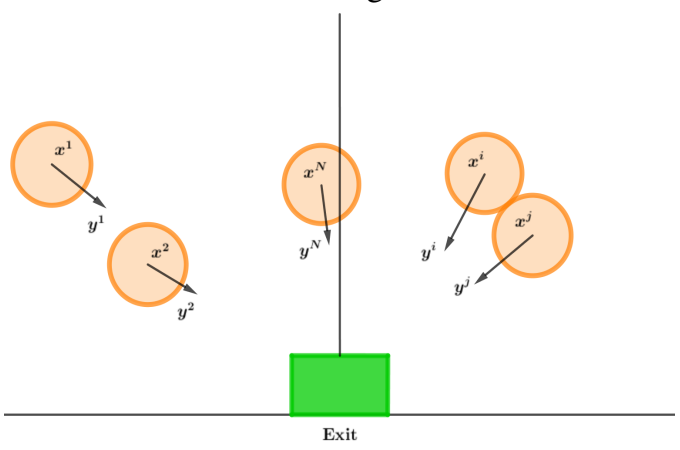

Fig. 1. Crowd motion model for $N$ participants

To formalize the ideas stated above, for $i=1, \ldots, N$, we denote by $\left(P_{H}\left(x_{0}, u\right)\right)$ the upper level problem in $\mathbb{R}^{2}$ :

$$
\begin{array}{cl}
\left(P_{H}\left(x_{0}, u\right)\right) & \text { Minimize } J_{H}\left(y ; x_{0}, u\right) \\
\text { subject to } & \dot{y}^{i}(t)=v^{i}(t) \quad[0, T] \text {-a.e., }, y^{i}(0)=y_{0}^{i} \\
& v^{i} \in \mathcal{V}^{i}:=\left\{v^{i} \in L^{2}\left([0, T] ; \mathbb{R}^{2}\right): v^{i}(t) \in V^{i}\right\} \\
& \left\|y^{i}(t)-y^{j}(t)\right\| \geq 2 R, j \neq i \forall t \\
& \text { and } y^{i} \text { s.t. } \exists \text { solution }\left(x_{0}^{i}, u^{i}\right) \text { to } P_{L}^{i}\left(y^{i}\right)(2)
\end{array}
$$

with $\left(y, x_{0}, u\right)^{T} \in \mathbb{R}^{N(4+m)}, a=\operatorname{col}\left\{a^{i}\right\}_{i=1}^{N}$, “col” stands for "column", and $J_{H}\left(y ; x_{0}, u\right):=\frac{1}{2} \sum_{i=1}^{N}\left\|y^{i}(T)\right\|^{2} . T$, and $y_{0}^{i}$ are fixed, and for $i, j=1, \ldots, N$ with $j \neq i,\left\|y_{0}^{i}-y_{0}^{j}\right\| \geq 2 R$, $\|\cdot\|$ is the Euclidean norm in $\mathbb{R}^{2} . V^{i} \subset \mathbb{R}^{2}$ stands for the control set and is compact. Condition (1) represents the nonoverlapping imposed on the translated disks $D^{i}(t):=D+y^{i}(t)$, $D^{j}(t):=D+y^{j}(t)$ with $D=\left\{x \in \mathbb{R}^{2}:\|x\| \leq R\right\}$, i.e., the distance between the centers of two disks have to be not less than $2 R$.

The problem $\left(P_{L}^{i}\left(y^{i}\right)\right)$, alluded to in 2, is defined by the following parametric lower level problem with dynamics 
involving a sweeping process with a controlled drift.

$$
\begin{aligned}
\left(P_{L}^{i}\left(y^{i}\right)\right) & \text { Minimize } J_{L}^{i}\left(x_{0}^{i}, u^{i} ; y^{i}\right) \\
\text { subject to } & \dot{x}^{i}(t) \in f^{i}\left(x^{i}(t), u^{i}(t)\right)-N_{D+y^{i}(t)}^{M^{i}}\left(x^{i}(t)\right) \\
& x^{i}(0)=x_{0}^{i} \in D+y_{0}^{i} \\
& u^{i}(t) \in \mathcal{U}^{i}:=\left\{u^{i} \in L^{\infty}\left([0, T] ; \mathbb{R}^{m}\right): u^{i}(t) \in U^{i}\right\} \\
& x^{i}(t) \in D+y^{i}(t) \quad \forall t \in[0, T],
\end{aligned}
$$

)

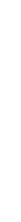

where, for a given process $y$ solving $\left(P_{H}\left(x_{0}, u\right)\right)$, $J_{L}^{i}\left(x_{0}^{i}, u^{i} ; y^{i}\right):=\int_{0}^{T}\left\|u^{i}(t)\right\|^{2} d t$ (control effort of each disk population), $f^{i}: \mathbb{R}^{2} \times \mathbb{R}^{m} \rightarrow \mathbb{R}^{2}, U^{i} \subset \mathbb{R}^{m}$ is compact, the truncated cone $N_{A^{i}}^{M^{i}}(z):=N_{A^{i}}(z) \cap M^{i} B_{1}(0)$, being $N_{A^{i}}(z)$ the Mordukhovich (limiting) normal cone to the closed set $A^{i}$ at point $z$ in the sense of [4], $B_{1}(0)$ the closed unit ball in $\mathbb{R}^{2}$ centered at the origin, and $M^{i}>0$ a given constant.

Let us define some concepts. Let $\left(P_{L}\right):=\operatorname{col}\left\{\left(P_{L}^{i}\right)\right\}_{i=1}^{N}$. For some parameter $y$, a pair $(x(\cdot), u(\cdot))$ is a feasible (or admissible) control process to $\left(P_{L}\right)$ if $u(\cdot)$ is feasible control to $\left(P_{L}\right)$, and $x(\cdot)$ is an arc satisfying the differential inclusion (3), the initial condition, together with (4). An optimal solution to $\left(P_{L}\right)$ is a feasible pair of $\left(P_{L}\right)$ minimizing the value of the cost functional $J_{L}\left(x_{0}, u ; y\right)$ over all admissible pairs of $\left(P_{L}(y)\right)$. A feasible triple of the dynamic control problem $\left(P_{H}\right)$ is the set of feasible process $y$, and an optimal pair $\left(x_{0}, u\right)$ to $\left(P_{L}(y)\right)$. The feasible triple $\left(y ; x_{0}, u\right)$ is optimal to $\left(P_{H}\right)$ if $\left(y ; x_{0}, u\right)$ minimizes the value of $J_{H}\left(y ; x_{0}, u\right)$ among all admissible strategies of $\left(P_{H}\right)$.

It is important to note that the articulation of $\left(P_{H}\right)$, and $\left(P_{L}\right)$ in this article features significant differences with respect to the bilevel control sweeping process problem studied in [1], and in [2]. Here, the upper level problem $\left(P_{H}\right)$ acts on the dynamics of each lower level sweeping control problems $\left(P_{L}^{i}\right)$, via the $\operatorname{arcs} y^{i}(\cdot)$ specifying the motion of the respective disks $D$. While the upper level problem minimizes the sum of distances of all the disk centers to the exit at the final time, and forbids their overlap, the lower level problem minimizes the control effort spent by any disk population in order to remain within the constraint set $\Pi_{1}^{N} D+y(t)$. Here $y$ is a feasible arc to $\left(P_{H}\right)$, and a parameter to $\left(P_{L}\right)$, and $\Pi_{1}^{N} D$ denotes the Cartesian product of $N$ disks $D$. We establish necessary conditions of optimality for this problem, and use them to solve an example with $N=2$.

In addition to the fact that a time-optimal problem is considered at the upper level instead of the minimal-path studied in the current article, in [1], the $N$ disks are confined in a larger constraint set, and $y^{i}, i=1, \ldots, N$ can take values on the boundary of this extra constraint set, giving rise to a sweeping process at the upper level. Only well-posedness and the existence of solutions to the problem are studied in [1]. However, in [2], a simpler instance of the problem n [1] is studied as only one disk (i.e. $N=1$ ) intervenes, and no sweeping process appears at the upper level problem. Necessary conditions of optimality are established for this problem.
The problem in [3] is of a different nature as it considers only a single-level (and not a bilevel) control sweeping problem. Another main difference is the nature of the sweeping process arising in the dynamics. Indeed, while in our paper the sweeping process appears, at the lower level problem, to force the various populations, with (average) motion velocity $\dot{x}^{i}(t)$ to stay confined to their moving set $D+y^{i}(t)$, in [3] the sweeping process intervenes to adjust velocities when a contact occurs between the disks, supposed to remain at a minimum distance from each other, avoiding overlapping scenarios. On the other hand, the technique used to deal with the sweeping process in [3] relies on the discrete approximation, while in our paper, we adopt another technique by approximating the sweeping term by a sequence of Lipschitz functions in the state variable. More details can be found in [2], and in Section IV of this paper.

Notation. We denote by $N_{A}(x)$ the Mordukhovich (limiting) normal cone to a closed set $A$ at the point $x \in A$, and by $\partial \varphi$, and $\partial^{C} \varphi$, respectively, the Mordukhovich, and the Clarke subdifferentials of $\varphi$. If $\varphi$ is locally Lipschitz, then $\partial^{C} \varphi=\operatorname{co} \partial \varphi$, where "co $A$ " denotes the closure of the convex hull of the set $A$. We refer the reader to [4], [5], [6] for a full overview on nonsmooth analysis. $A C\left([0, T] ; \mathbb{R}^{2 N}\right)$ stands for the space of absolutely continuous functions on $[0, T]$ with values in $\mathbb{R}^{2 N}, B V\left([0, T] ; \mathbb{R}^{+}\right)$for the space of functions of bounded variations on $[0, T]$ with nonnegative values, $\|\cdot\|_{T V}$ the total variation, and superscript $T$ the transpose. Given $a \in \mathbb{R}^{k}$, and $b \in \mathbb{R}^{k m}$, we have $a \diamond b=$ $\operatorname{col}\left(a^{i}\left(b^{(i-1) m+1}, \cdots, b^{i m}\right)^{T}: i=1, \ldots, k\right)$.

The article is organized as follow: in Section III, we give the required assumptions to be imposed on the data. In Section [III. we establish the necessary optimality condition of the problem setting $\left(P_{H}\right)-\left(P_{L}\right)$. A proof outline with the key ideas is given in Section IV An example considering the case of only two disks is studied in Section $\mathrm{V}$. We finish with a conclusion and some remarks for future avenues.

\section{ASSUMPTIONS}

Before stating the necessary optimality conditions, we present the assumptions to be imposed on the data of the problem. These are as follows, for all $i=1, \ldots, N$ :

H1 $f^{i}\left(x^{i}, \cdot\right)$ is Borel measurable $\forall x^{i} \in \mathbb{R}^{2}, f^{i}\left(\cdot, u^{i}\right)$ is Lipschitz continuous $\forall u^{i} \in U^{i}$, and $f^{i}(\cdot, \cdot)$ is bounded $\forall\left(x^{i}, u^{i}\right) \in \mathbb{R}^{2} \times U^{i}$.

H2 $f^{i}\left(x^{i}, U^{i}\right) \subset \mathbb{R}^{2}$ is a closed and convex set for each $x^{i}$.

H3 The control sets $U^{i}$, and $V^{i}$ are compact and convex.

H4 There exists $\beta>0$ s.t. $\beta B_{1}(0) \subset f^{i}\left(x^{i}, U^{i}\right), \forall x^{i} \in \mathbb{R}^{2}$.

H5 The constant $M^{i}$ specifying the truncation of the normal cone satisfies $\bar{M}^{i}>M^{i}>\bar{m}^{i}$ where, $\forall \zeta^{i} \in$ $N_{D+y^{i}(t)}\left(x^{i}(t)\right), \forall t \in[0, T]$ with $x^{i}(t) \in \operatorname{bd}\left(D+y^{i}(t)\right)$ ("bd" is the boundary),

$$
\begin{aligned}
& \bar{M}^{i}=\min _{\left\|\zeta^{i}\right\|=1}\left\{\max _{u \in U^{i}}\left\{\left\langle\zeta^{i}, f^{i}\left(x^{i}(t), u\right)\right\rangle\right\}-\min _{v \in V^{i}}\left\{\left\langle\zeta^{i}, v\right\rangle\right\},\right. \\
& \bar{m}^{i}=\max _{\left\|\zeta^{i}\right\|=1}\left\{\min _{u \in U^{i}}\left\{\left\langle\zeta^{i}, f^{i}\left(x^{i}(t), u\right)\right\rangle\right\}-\max _{v \in V^{i}}\left\{\left\langle\zeta^{i}, v\right\rangle\right\}\right\} .
\end{aligned}
$$


We also require additional assumptions playing a critical role in the articulation of $\left(P_{H}\right)$, and $\left(P_{L}\right)$, and in the derivation of the necessary conditions of optimality. Similar assumptions has been cpnsidered in [7], [8], albeit for in a different context:

H6 For a given $y$ feasible to $\left(P_{H}\right)$, the solution set of $\left(P_{L}\right)$ is not empty and every process $(x, u)$ solving $\left(P_{L}\right)$ lies in the interior of its solution set.

H7 The articulation of $\left(P_{H}\right)-\left(P_{L}\right)$ is partially calm, i.e. , $\exists \rho^{i} \geq 0$ such that for any feasible $(y, x, v, u)$,

$$
\begin{aligned}
J_{H}\left(y ; x_{0}, u\right)-J_{H}\left(y^{*} ; x_{0}^{*}, u^{*}\right) & \\
& +\sum_{i=1}^{N} \rho^{i}\left(\int_{0}^{T}\left\|u^{i}(s)\right\|^{2} d s-\varphi^{i}\left(v^{i}\right)\right) \geq 0,
\end{aligned}
$$

where $\left(y^{*}, x_{0}^{*}, u^{*}\right)$ is an optimal solution to $\left(P_{H}\right)-\left(P_{L}\right)$, and $\varphi^{i}(\cdot)$ is the value function of $\left(P_{L}^{i}\right)$ defined by

$$
\begin{gathered}
\varphi^{i}\left(v^{i}\right)=\min \left\{J_{L}^{i}\left(x_{0}^{i}, u^{i} ; y^{i}\right):\left(x_{0}^{i}, u^{i}\right) \text { feasible for }\left(P_{L}^{i}\right)\right. \\
\text { and } \left.y^{i}(t)=y_{0}^{i}+\int_{0}^{t} v^{i}(s) d s\right\}
\end{gathered}
$$

being $v^{i}$ the $i^{t h}$ component of $v$, an admissible to $\left(P_{H}\right)$.

\section{NECESSARY OPTIMALITY CONDITIONS}

Before stating the necessary optimality conditions of $\left(P_{H}\right)-\left(P_{L}\right)$, we shall first define the following

$$
\begin{aligned}
H_{H} & \left(y, x, v, u, q_{H}, q_{L}, \nu_{H}, \nu_{L}, \alpha\right) \\
& =\sum_{i=1}^{N} H_{H}^{i}\left(y^{i}, x^{i}, v^{i}, u^{i}, q_{H}^{i}, q_{L}^{i}, \bar{\nu}_{H}^{i}, \nu_{L}^{i}, \alpha^{i}\right)
\end{aligned}
$$

where $y, x, v, u, q_{H}, q_{L}$ take values in $\mathbb{R}^{2 N}, \nu_{L}$ are in $\mathbb{R}_{+}^{N}$, $\nu_{H} \in \mathbb{R}_{+}^{N(N-1)}, \bar{\nu}_{H}^{i} \in \mathbb{R}_{+}^{N-1}$ is a vector with components $\nu_{H}^{i j}$ satisfying $j \neq i$, and $\nu_{H}^{i j}=\nu_{H}^{j i}$ (symmetric), and for a fixed $i \in\{1, \ldots, N\}$, we have

$$
\begin{aligned}
H_{H}^{i}\left(y^{i}, x^{i}, v^{i}, u^{i}, q_{H}^{i}, q_{L}^{i}, \bar{\nu}_{H}^{i}, \nu_{L}^{i}, \alpha^{i}\right) \\
:=\left\langle q_{L}^{i}-\nu_{L}^{i}\left(x^{i}-y^{i}\right), f^{i}\left(x^{i}, u^{i}\right)\right\rangle+\nu_{L}^{i}\left\langle x^{i}-y^{i}, v^{i}\right\rangle \\
\quad+\sigma^{i}\left(y^{i}, x^{i}, q_{L}^{i}, \nu_{L}^{i}\right)-\alpha^{i}\left\|u^{i}\right\|^{2} \\
+\left\langle q_{H}^{i}+\sum_{j<i}^{N} \nu_{H}^{i j} \frac{y^{i}-y^{j}}{\left\|y^{i}-y^{j}\right\|}+\sum_{j>i}^{N} \nu_{H}^{i j} \frac{y^{i}-y^{j}}{\left\|y^{i}-y^{j}\right\|}, v^{i}\right\rangle,
\end{aligned}
$$

where $\sigma^{i}\left(y^{i}, x^{i}, q_{L}^{i}, \nu_{L}^{i}\right)=\sup _{\xi \in-N_{D^{i}}^{M^{i}}\left(x^{i}-y^{i}\right)}\left\{\left\langle q_{L}^{i}-\nu_{L}^{i}\left(x^{i}-y^{i}\right), \xi\right\rangle\right\}$.

We note that the multiplier $\bar{\nu}_{H}^{i}=\left\{\nu_{H}^{i j}\right\}_{i \neq j}$ appears in $H_{H}^{i}$ to reflect the activity of the constraint $\left\|y^{i} y^{j}\right\| \geq 2 R$ (i.e., , 11 ). It is non-increasing whenever $\left\|y^{i}-y^{j}\right\|=2 R$ (i.e., the disks $D+y^{i}(t)$ and $D+y^{j}(t)$ are in contact), and constant otherwise.

The form of the Hamiltonian $H_{H}$ stated above is different from the usual Pontryagin-Hamilton function used in the Dubovitskii-Milyutin form [9]. Our Hamiltonian is the one used in establishing the necessary conditions of optimality in the Gamkrelidze's form [10], [11]. These results, and further developments were recently incorporated in the modern optimal control literature in [12], [13], [14]. This form differs from the Dubovitskii-Milyutin one in the way the measure multiplier associated with the state constraints enters in the Pontryagin-Hamiltonian function. It might entail some loss of generality due to the extra smoothness required on the function specifying the state constraints, but opens significant new computational perspectives for indirect methods based on the Maximum Principle of Pontryagin due to the regularity of its measure multiplier.

Theorem 3.1: Let $\mathrm{H} 1-\mathrm{H} 7$ hold and $\left(y^{*}, x^{*}, u^{*}\right)$ a solution to $\left(P_{H}\right)-\left(P_{L}\right)$. Then, there exists a set of multipliers $\left(q_{H}, q_{L}, \nu_{H}, \nu_{L}, \lambda, \alpha\right)$ with $q_{H}$, and $q_{L}$ in $A C\left([0, T] ; \mathbb{R}^{2 N}\right)$, $\nu_{H} \in B V\left([0, T] ; \mathbb{R}_{+}^{N(N-1)}\right)$, and $\nu_{L} \in B V\left([0, T] ; \mathbb{R}_{+}^{N}\right)$ being both non-increasing, and $\nu_{H}^{i j}$, and $\nu_{L}$ constants on $\{t \in[0, T]$ : $\left.\left\|y^{i}-y^{j}\right\|>2 R, j \neq i\right\}$, and $\{t \in[0, T]:\|y-x\|<R\}$, respectively, and $\lambda \in[0,1], \alpha \in \mathbb{R}^{N}$, with $\alpha^{i}=\lambda \rho^{i}, i=$ $1, \ldots, N$ (being $\rho^{i}$ the modulus in $\left.(\mathrm{H} 7)\right)$, with:

1. Nontriviality. $\left\|\left(q_{H}, q_{L}\right)\right\|_{L^{\infty}}+\left\|\left(\nu_{H}, \nu_{L}\right)\right\|_{T V}+\lambda+|\alpha| \neq 0$

2. Adjoint equations.

$$
\begin{array}{r}
-\dot{q}_{L}(t) \in \partial_{x} H_{H}\left(y^{*}, x^{*}, v^{*}, u^{*}, q_{H}, q_{L}, \nu_{H}, \nu_{L}, \alpha\right) \\
=\partial_{x}\left\langle q_{L}(t)-\nu_{L}(t) \diamond\left(x^{*}(t)-y^{*}(t)\right), f^{*}(t)\right\rangle \\
+\nu_{L}(t) v^{*}(t)+\partial_{x} \sigma\left(y^{*}(t), x^{*}(t), q_{L}(t), \nu_{L}(t)\right) \text { a.e. } \\
-\dot{q}_{H}(t) \in \partial_{y} H_{H}\left(y^{*}, x^{*}, v^{*}, u^{*}, q_{H}, q_{L}, \nu_{H}, \nu_{L}, \alpha\right) \\
=-\nu_{L}(t) \diamond v^{*}(t)+\nu_{L}(t) \diamond f^{*}(t) \\
+\partial_{y} \sigma\left(y^{*}(t), x^{*}(t), q_{L}(t), \nu_{L}(t)\right) \\
+\operatorname{col}\left(\sum_{j>1} \nu_{H}^{1 j}(t) d^{1 j}(t) v^{1 *}(t)\right. \\
\ldots ; \sum_{j<i} \nu_{H}^{i j}(t) d^{i j}(t) v^{i *}(t) \\
+\sum_{j>i} \nu_{H}^{i j}(t) d^{i j}(t) v^{i *}(t) \\
\left.\ldots ; \sum_{j<N} \nu_{H}^{N j}(t) d^{N j}(t) v^{N *}(t)\right) \text { a.e. }
\end{array}
$$

being $d^{i j}:=\left\|y^{i *}-y^{j *}\right\|^{-1} I-\frac{\left(y^{i *}-y^{j *}\right)\left(y^{i *}-y^{j *}\right)^{T}}{\left\|y^{i *}-y^{j *}\right\|^{3}}, I$ the unit matrix, $f^{*}(t):=f\left(x^{*}(t), u^{*}(t)\right)$, and $\sigma=\sum_{i=1}^{N} \sigma^{i}$.

3 . Boundary conditions.

$$
\begin{array}{r}
q_{H}(0) \in \mathbb{R}^{2 N} \\
q_{L}(0) \in N_{D+y_{0}}\left(x^{*}(0)\right)+\nu_{L}(0) \diamond\left(x^{*}(0)-y_{0}\right) \\
q_{H}(T)=-\lambda y^{*}(T)-\nu_{L}(T) \diamond\left(x^{*}(T)-y^{*}(T)\right) \\
-\operatorname{col}\left(\sum_{j>1} \nu_{H}^{1 j}(T) \frac{y^{1 *}(T)-y^{j *}(T)}{\left\|y^{1 *}(T)-y^{j *}(T)\right\|} ;\right. \\
\ldots ; \sum_{j<i} \nu_{H}^{i j}(T) \frac{y^{i *}(T)-y^{j *}(T)}{\left\|y^{i *}(T)-y^{j *}(T)\right\|} \\
+\sum_{j>i} \nu_{H}^{i j}(T) \frac{y^{i *}(T)-y^{j *}(T)}{\left\|y^{i *}(T)-y^{j *}(T)\right\|} \\
\ldots ; \sum_{j<N} \nu_{H}^{N j}(T) \frac{y^{N *}(T)-y^{j *}(T)}{\left\|y^{N *}(T)-y^{j *}(T)\right\|}
\end{array}
$$


4. Maximum condition on the lower level control. $u^{*}(t)$ maximizes on $U=U^{1} \times \ldots \times U^{N},[0, T]$-a.e.

$$
\begin{aligned}
\left(u^{1}, \ldots,\right. & \left.u^{N}\right) \rightarrow-\sum_{i=1}^{N} \alpha^{i}\left\|u^{i}\right\|^{2} \\
& +\left\langle q_{L}(t)-\nu_{L}(t) \diamond\left(x^{*}(t)-y^{*}(t)\right), f\left(x^{*}(t), u\right)\right\rangle .
\end{aligned}
$$

5. Maximum condition on the upper level control.

$$
\begin{gathered}
q_{H}(t)+\nu_{L}(t) \diamond\left(x^{*}(t)-y^{*}(t)\right) \\
+\operatorname{col}\left(\sum_{j>1} \nu_{H}^{1 j}(t) \frac{y^{1 *}(t)-y^{j *}(t)}{\left\|y^{1 *}(t)-y^{j *}(t)\right\|} ;\right. \\
\ldots ; \sum_{j<i} \nu_{H}^{i j}(t) \frac{y^{i *}(t)-y^{j *}(t)}{\left\|y^{i *}(t)-y^{j *}(t)\right\|} \\
+\sum_{j>i} \nu_{H}^{i j}(t) \frac{y^{i *}(t)-y^{j *}(t)}{\left\|y^{i *}(t)-y^{j *}(t)\right\|} ; \\
\left.\ldots ; \sum_{j<N} \nu_{H}^{N j}(t) \frac{y^{N *}(t)-y^{j *}(t)}{\left\|y^{N *}(t)-y^{j *}(t)\right\|}\right) \\
\in \prod_{i=1}^{N} \alpha^{i} \partial_{v^{i}}^{C} \varphi^{i}\left(v^{i *}(t)\right)-\prod_{i=1}^{N} N_{\mathcal{V}^{i}}\left(v^{i *}(t)\right) .
\end{gathered}
$$

Here, each $\partial_{v^{i}}^{C} \varphi^{i}\left(v^{i *}(t)\right), i=1, \ldots, N$, is defined as below.

For $i=1, \ldots, N$, denote by $\Psi^{i}\left(y^{i}\right)$ the sets of optimal solutions to the lower level problems $\left(P_{L}^{i}\right)$, and by $H_{L}^{i}$ the Pontryagin-Hamiltonian functions given by

$$
\begin{aligned}
H_{L}^{i}:= & \mu_{L}^{i}\left\langle x^{i}-y^{i}, v^{i}\right\rangle+\sup _{z \in-N_{D^{i}+y^{i}}}\left\{\left\langle x^{i}\right)\right. \\
& \left.\left.+\sup _{L}^{i}-\mu_{L}^{i}\left(x^{i}-y^{i}\right), z\right\rangle\right\} \\
& \left\{\left\langle p_{L}^{i}-\mu_{L}^{i}\left(x^{i}-y^{i}\right), f^{i}\left(x^{i}, u^{i}\right)\right\rangle-\bar{\lambda}^{i}\left\|u^{i}\right\|^{2}\right\} \\
+ & \left\langle p_{H}^{i}+\sum_{j<i} \mu_{H}^{i j} \frac{y^{i}-y^{j}}{\left\|y^{i}-y^{j}\right\|}+\sum_{j>i} \mu_{H}^{i j} \frac{y^{i}-y^{j}}{\left\|y^{i}-y^{j}\right\|}, v^{i}\right\rangle
\end{aligned}
$$

for $y^{i}, x^{i}, v^{i}, p_{H}^{i}, p_{L}^{i}$ in $\mathbb{R}^{2}, u^{i} \in \mathbb{R}^{2}, \mu_{H}^{i j}, \mu_{L}^{i}, \bar{\lambda}^{i} \in \mathbb{R}_{+}$. Then, we have

$$
\partial_{v^{i}}^{C} \varphi^{i}\left(v^{i *}\right):=\operatorname{co} \bigcup_{x^{i} \in \Psi^{i}\left(y^{i *}\right)}\left\{\zeta^{i} \in L^{2}\left([0, T]: \mathbb{R}^{2}\right):\left(\mathcal{A}^{i}\right) \text { holds }\right\} .
$$

The relation $\left(\mathcal{A}^{i}\right)$ is defined as follow (the dependence on $t$ is discarded for simplicity): there exists $\left(p_{H}^{i}, p_{L}^{i}, \mu_{H}^{i j}, \mu_{L}^{i}, \bar{\lambda}^{i}\right) \in$ $A C \times A C \times B V \times B V \times \mathbb{R}_{+}$such that

a) $\left\|\left(p_{H}^{i}, p_{L}^{i}\right)\right\|_{L^{\infty}}+\left\|\left(\mu_{H}^{i j}, \mu_{L}^{i}\right)\right\|_{T V}+\bar{\lambda}^{i} \neq 0$.

b) $\mu_{H}^{i j}, \mu_{L}^{i}$ are non-increasing, and constant, respectively, on $\left\{t \in[0, T]:\left\|y^{i}-y^{j}\right\|>2 R, i \neq j, i, j=1, \ldots, N\right\}$ and $\left\{t \in[0, T]:\left\|y^{i}-x^{i}\right\|<R\right\}$.

c) $\left(\dot{y}^{i}, \dot{x}^{i},-\dot{p}_{H}^{i},-\dot{p}_{L}^{i}\right) \in \partial_{\left(p_{H}^{i}, p_{L}^{i}, y^{i}, x^{i}\right)}^{C} H_{L}^{i}$ a.e.

d) $\bar{\lambda}^{i} \zeta^{i}+p_{H}^{i}+\mu_{L}^{i}\left(x^{i}-y^{i}\right)$

$$
+\sum_{j<i} \mu_{H}^{i j} \frac{y^{i}-y^{j}}{\left\|y^{i}-y^{j}\right\|}+\sum_{j>i} \mu_{H}^{i j} \frac{y^{i}-y^{j}}{\left\|y^{i}-y^{j}\right\|} \in-N_{\mathcal{V}^{i}}\left(v^{i}\right) .
$$

e) $p_{H}^{i}(0) \in \mathbb{R}^{2}$,

$$
p_{L}^{i}(0) \in N_{D+y_{0}^{i}}\left(x^{i}(0)\right)+\mu_{L}^{i}(0)\left(x^{i}(0)-y_{0}^{i}\right),
$$

$$
\begin{aligned}
& p_{L}^{i}(T)=\mu_{L}^{i}(T)\left(x^{i}(T)-y^{i}(T)\right), \\
& p_{H}^{i}(T)=-\mu_{L}^{i}(T)\left(x^{i}(T)-y^{i}(T)\right) \\
& \quad-\sum_{j<i} \mu_{H}^{i j}(T) \frac{y^{i}(T)-y^{j}(T)}{\left\|y^{i}(T)-y^{j}(T)\right\|} \\
& \quad-\sum_{j>i} \mu_{H}^{i j}(T) \frac{y^{i}(T)-y^{j}(T)}{\left\|y^{i}(T)-y^{j}(T)\right\|} .
\end{aligned}
$$

\section{BRIEF OUTLINE OF THE KEY IDEAS OF THE PROOF}

The two main obstacles encountered when dealing with a bilevel control sweeping problem in the form of $\left(P_{H}\right)$ $\left(P_{L}\right)$ is first the discontinuity of the normal cone w.r.t. the state variable, and second the extra boundary constraint (2). Indeed, the normal cone, being part of the dynamics of the lower level problem, lacks the Lipschitz property (with respect to the state variable) crucial to establish the standard necessary optimality condition. On the other hand, in the presence of condition (2), the constraint qualifications, such as Mangasarian-Fromovitz or linear independence constraint qualifications, are likely to be violated and, thus, entailing a degeneracy phenomena.

In order to encounter these two challenges, we base the proof of Theorem 3.1 mainly on two key pillars: the smooth approximation of the truncated normal cone by a sequence of Lipschitz functions in the state, and the flattening of the bilevel structure, under the partial calmness condition, by penalizing the cost of the upper level problem with the problematic term, i.e. the value function $\varphi$ representing condition (2). The main ideas of the proof are based on the ones in [2], however, adapted to our context, mainly in what concerns the consideration of $N \geq 2$ participating disks and the non-overlapping between them, while in [2], only one disk is considered and therefore no overlapping is involved.

\section{CROWD MOTION EXAMPLE}

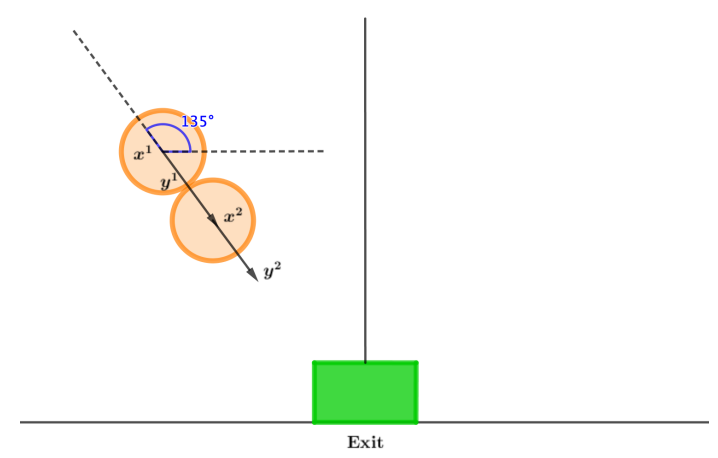

In this example and to avoid confusion, we adopt the subscript notation when referring to the dynamics, controls, trajectories, and the state constraints, while keeping the superscript notation for the multipliers and the Hamiltonians.

We take two disks $D_{i}, i=1,2$ of the same radius $R=3$. At the upper level problem, given a fixed final time $T=6$, we consider the controls $v_{1}$, and $v_{2}$ taking values in $V_{i}=$ $\left\{a\left(\cos \left(\theta_{i}\right), \sin \left(\theta_{i}\right)\right)^{T}: a \in[-\mathbf{V}, \mathbf{V}]\right\}$, with $\mathbf{V}=10 \sqrt{2}$. Vectors $y_{1}(t)$, and $y_{2}(t)$ defining the linear directions of disks, 
respectively, $D_{1}$, and $D_{2}$, satisfy the following dynamics

$$
\begin{gathered}
\dot{y}_{1}(t)=v_{1}=-\bar{v}_{1}\left(\cos \theta_{1}, \sin \theta_{1}\right), \\
\dot{y}_{2}(t)=v_{2}=-\bar{v}_{2}\left(\cos \theta_{2}, \sin \theta_{2}\right),
\end{gathered}
$$

where $\theta_{1}$, and $\theta_{2}$ are the smallest positive angles formed by the positive $x$-axis with $y_{1}$, and $y_{2}$, respectively.

The disks keep the same direction until the final time, which entails that $\dot{y}_{1}(t)=\dot{y}_{2}(t)$ for all $t \in[0,6]$, and, thus, $\bar{v}_{1}=\bar{v}_{2}$.

At the upper level, we minimize the sum of the distances of the two disks to the exit at the origin:

$$
\min J_{H}:=\frac{1}{2}\left(\left\|y_{1}(6)\right\|^{2}+\left\|y_{2}(6)\right\|^{2}\right) \text {. }
$$

Consider $y_{1}^{0}=(-48-3 \sqrt{2}, 48+3 \sqrt{2})^{T}, y_{2}^{0}=\left(-48,48^{T}\right)$, and $\theta_{1}=\theta_{2}=135^{\circ}$. In this case, at the initial time $t=0$, the two disks are in contact (indeed $\left\|y_{1}^{0}-y_{2}^{0}\right\|=2 R=6$ ), and $\left(\cos \theta_{i}, \sin \theta_{i}\right)=\mathbf{v}$, for $i=1,2$, where $\mathbf{v}=\left(-\frac{\sqrt{2}}{2}, \frac{\sqrt{2}}{2}\right)^{T}$.

At the lower level, we consider two scalar control functions $u_{i}(\cdot), i=1,2$ taking values in $U_{i}=[0,1]$ corresponding, to the efforts of the population of disks $D_{i}+y_{i}$. In each disk, the population is represented by its position $x_{i}, i=1,2$. Denote by $t_{a}$ the contact time of $x_{i}$ with the boundary of the moving disks $D_{i}+y_{i}$. Moreover, once the population hits the boundary of the corresponding moving disk, it does not leave the boundary until the final time. The (average) dynamics of the populations are, for all $t \in[0,6]$ :

$$
\begin{aligned}
& \dot{x}_{1}(t)=-8 u_{1}(t) x_{1}(t)-\beta_{1}(t) u_{0}\left(x_{1}(t)-y_{1}(t)\right) \\
& \dot{x}_{2}(t)=-8 u_{2}(t) x_{2}(t)-\beta_{2}(t) u_{0}\left(x_{2}(t)-y_{2}(t)\right)
\end{aligned}
$$

being

$$
\beta_{1}(t)=\left\{\begin{array}{ll}
\frac{M_{1}}{3} & \forall t \in\left[t_{a}, 6\right] \\
0 & \forall t \in\left[0, t_{a}\right)
\end{array}, \beta_{2}(t)= \begin{cases}\frac{M_{2}}{3} & \forall t \in\left[t_{a}, 6\right] \\
0 & \forall t \in\left[0, t_{a}\right),\end{cases}\right.
$$

$u_{0} \in[0,1]$ a control, and $M_{1}>0$, and $M_{2}>0$ are such that H5 is satisfied. Thus, we let $M_{1}=M_{2}=6$.

The objective functions to minimize at the lower level are the control efforts of the populations to stay confined to their moving disks $D_{1}+y_{1}$ and $D_{2}+y_{2}$

$$
\min \int_{0}^{6}\left\|u_{1}(t)\right\|^{2} d t, \quad \min \int_{0}^{6}\left\|u_{2}(t)\right\|^{2} d t .
$$

Consider also for simplicity $\left(x_{1}^{0}, x_{2}^{0}\right)=\left(y_{1}^{0}, y_{2}^{0}\right)$, meaning that at the initial time $t=0$, each population is in the interior of its disk.

As follows from the problem formulation, the leader/follower solution concept entails the fact that the lower level problems relations constitute constraints to the upper level problem and there is no relation order on the lower level performance values. This, together with the geometric insight dictate that $D_{1}$ and $D_{2}$ are driven from their initial position by the upper level dynamics along the line $(\alpha \mathbf{v}: \alpha \in \mathbb{R}\}$ to a final time position minimizing $J_{H}$ while enabling the lower level control problem constraints to be satisfied. On the other hand, the lower level controls should be such that $x_{i}^{*}$ stays as much as possible on the boundary of the disks $D_{i}+y_{i}^{*}, i=1,2$. It is straightforward to conclude that $v_{1}^{*}(t)=v_{2}^{*}(t) \forall t \in[0,6]$ and that $\exists t_{a}, t_{b}$ such that $0<t_{a}<t_{b}<6$, and $\bar{v}^{*}>0$ so that the optimal controls are

$$
\begin{array}{r}
v_{i}^{*}(t)= \begin{cases}-\bar{v}^{*} \mathbf{v} & \text { if } t \in\left[0, t_{b}\right] \\
-\left(8 \gamma_{2}(t)+6\right) \mathbf{v} & \text { if } t \in\left[t_{b}, 6\right]\end{cases} \\
u_{i}^{*}(t)= \begin{cases}0 & \text { if } t \in\left[0, t_{a}\right] \\
\frac{\bar{v}^{*}-6}{8\left\|x_{i}^{*}(t)\right\|} & \text { if } t \in\left[t_{a}, t_{b}\right)\end{cases} \\
\forall t \in\left[t_{b}, 6\right],\left\{\begin{array}{l}
u_{1}^{*}(t)=\frac{\left\|x_{2}^{*}(t)\right\|}{\left\|x_{2}^{*}(t)\right\|+6} \\
u_{2}^{*}(t)=1
\end{array}\right.
\end{array}
$$

where $\gamma_{2}$ is such that $x_{2}^{*}(t)=\gamma_{2}(t) \mathbf{v}$ (note that $\left\|x_{2}^{*}\right\|=\gamma_{2}(t)$, whenever $\left.\gamma_{2} \geq 0\right)$. Observe that, for $i=1,2$,

- The value of $\bar{v}^{*}$ on $\left[t_{b}, 6\right]$ in $(7)$ is required because $u_{2}^{*}(t) \leq 1$, and $\dot{x}_{2}^{*}(t)=v_{2}^{*}(t), \forall t \in[0,6]$.

- In (8), the values of $u_{i}^{*}(t)$ on $\left[0, t_{a}\right]$ ensure that $x_{i}^{*}$ hits the boundary of $D_{i}+y_{i}^{*}$ as fast as possible, i.e., at $t=t_{a}$, which can be computed from $\bar{v}^{*} t_{a}=3$, and the ones in $\left(t_{a}, t_{b}\right]$, entails that $\dot{x}_{i}^{*}(t)=\dot{y}_{i}^{*}(t)$.

- The values of $u_{i}^{*}(t)$ on $\left[t_{b}, 6\right]$ in (9) reflect the upper bound on the values of $u_{i}^{*}$, and the relation $\dot{x}_{1}^{*}(t)=\dot{x}_{2}^{*}(t)$. Remark here that we always have $u_{1}^{*}(t)<1, \forall t \in[0,6]$, and that $t_{b}$ and $\bar{v}^{*}$ can be related by the second control value expression in $(8)$ for $i=2$, i.e., $\bar{v}^{*}=8\left\|x_{2}^{*}\left(t_{b}\right)\right\|+6$.

Now, we have that $\gamma_{2}(t)=48 \sqrt{2}+3-\bar{v}^{*} t$, and, by solving $\dot{\gamma}_{2}(t)=-8 \gamma_{2}-6$ on $\left[t_{b}, 6\right]$, we obtain, for this interval, $\gamma_{2}(t)=\frac{1}{8}\left(\bar{v}^{*} e^{-8\left(t-t_{b}\right)}-6\right)$. From the continuity of $\gamma_{2}$ at $t_{b}$, we find that $\frac{1}{8} \bar{v}^{*}\left(8 t_{b}+1\right)=48 \sqrt{2}+3.75$. Moreover, by integrating $\bar{v}_{2}^{*}$, the value of $y_{2}(6)$ is obtained. After eliminating $\bar{v}^{*}$ with the previous equality we conclude that

$$
y_{2}(6)=\left(-3.75+\frac{48 \sqrt{2}+3.750}{8 t_{b}+1} e^{-8\left(6-t_{b}\right)}\right) \mathbf{v} .
$$

By noting that $y_{1}^{*}(t)=y_{2}^{*}(t)+6 \mathbf{v}$, and, by optimizing $J_{H}$ on $t_{b}$ as a function of $t_{b}$ alone, we obtain $t_{b} \approx 5.915$, and, thus $\bar{v}^{*} \approx 11.860$, and $t_{a} \approx 0.253$.

Now, we show that the solution above satisfies the necessary optimality conditions of Theorem 3.1 We first notice that the assumptions stated in $\mathrm{H} 1-\mathrm{H} 7$ are satisfied. In what concerns checking the optimality conditions, we notice that there is a certain symmetry between the control processes $\left(y_{1}^{*}, v_{1}^{*}, x_{1}^{*}, u_{1}^{*}\right)$, and $\left(y_{2}^{*}, v_{2}^{*}, x_{2}^{*}, u_{2}^{*}\right)$ on the interval $\left[0, t_{b}\right]$. This symmetry is broken on $\left[t_{b}, 6\right]$ due to the fact while $u_{2}^{*}$ is on the boundary of its constraints set that is not the case for $u_{1}^{*}$. Thus, the brief outline of the verification of the optimality conditions will be made with respect to the former.

By expliciting the optimality conditions of Theorem 3.1 at the computed $\left(v_{1}^{*}, v_{2}^{*}, u_{1}^{*}, u_{2}^{*}\right)$, we notice that the optimal control at the upper level $\left(v_{1}^{*}, v_{2}^{*}\right)$ is indeed in the interior of $V_{1} \times V_{2}$, otherwise $\left(v_{1}, v_{2}\right) \in \operatorname{bd}\left(V_{1} \times V_{2}\right)$, equivalently, and either $v_{1}^{*}=v_{2}^{*}=0$ with the overall system at rest, or $v_{1}^{*}=v_{2}^{*}=10 \sqrt{2} \mathbf{v}$. In either case the control process is obviously not optimal. 
Then, the maximum condition 5. for the upper level problem yields

$$
\begin{aligned}
q_{H}^{2} & +\nu_{L}^{2}\left(x_{2}^{*}-y_{2}^{*}\right)+\nu_{H}^{21} \frac{y_{2}^{*}-y_{1}^{*}}{\left\|y_{2}^{*}-y_{1}^{*}\right\|} \\
& =-\frac{\alpha^{2}}{\bar{\lambda}^{2}}\left[p_{H}^{2}+\mu_{L}^{2}\left(x_{2}^{*}-y_{2}^{*}\right)+\mu_{H}^{21} \frac{y_{2}^{*}-y_{1}^{*}}{\left\|y_{2}^{*}-y_{1}^{*}\right\|}\right]
\end{aligned}
$$

which is a consequence of condition d) by remarking that

$$
\partial_{v_{2}} \varphi_{2}\left(v_{2}^{*}\right) \in\left\{-\frac{1}{\bar{\lambda}_{2}}\left[p_{H}^{2}+\mu_{L}^{2}\left(x_{2}^{*}-y_{2}^{*}\right)+\mu_{H}^{21} \frac{y_{2}^{*}-y_{1}^{*}}{\left\|y_{2}^{*}-y_{1}^{*}\right\|}\right]\right\} .
$$

A similar analysis can be made with $q_{H}^{1}, \nu_{L}^{1}, \mu_{L}^{1}, \partial_{v_{1}} \varphi_{1}\left(v_{1}^{*}\right)$.

In order to check whether the maximum condition is satisfied by the proposed optimal control $u_{2}^{*}$, we differentiate $H_{H}^{2}$ with respect to $u_{2}$, to obtain the expression

$$
-8 \bar{x}_{2}^{*}\left(\left\langle q_{L}^{2}, \mathbf{v}\right\rangle-\frac{1}{2} \nu_{L}^{2}\left\|x_{2}^{*}-y_{2}^{*}\right\|\right)-2 \alpha^{2} u_{2}^{*},
$$

where $\bar{x}_{2}^{*}$ is such that $x_{2}^{*}=\bar{x}_{2}^{*} \mathbf{v}$, By concatenating the various segments and taking into account the system and adjoint dynamics, their boundary conditions, including the one articulating both levels, the state constraints, and their associated multipliers, we conclude that there exist multipliers for which $\nabla H_{H}^{2} \leq 0$ on $\left[0, t_{a}\right]$ whenever $\left\|x_{2}^{*}-y_{2}^{*}\right\|<3, \nabla H_{H}^{2}=0$ on $\left[t_{a}, t_{b}\right]$ whenever $\left\|x_{2}^{*}-y_{2}^{*}\right\|=3$, being $H_{H}^{2}$ maximized by the proposed $u_{2}^{*}$, and $\nabla H_{H}^{2} \geq 0$ again when the low level state constraint is active, forcing $u_{2}^{*}=1$. Similar reasoning can be made for $u_{1}^{*}$.

Now, by evaluating (10) at the final time $T=6$, and assuming (without loss of generality) that all the measures at $T=6$ are equal to zero, we obtain, from the boundary conditions (condition 3.) and from $y_{1}^{*}(6) \neq 0$ (also $y_{2}^{*}(6) \neq$ 0 ) that $\lambda=0$ (yielding that $\alpha^{1}=\alpha^{2}=0$ ). By substituting again in (10), but now for a.e. $t \in\left[t_{a}, 6\right]$ and by taking the norm, we obtain that $\left\|\left(q_{H}^{1}, q_{H}^{2}\right)\right\|=\left\|\left(\nu_{L}^{1}, \nu_{L}^{2}\right)\right\|=\left\|\nu_{H}^{12}\right\|=0$. By replacing in the maximum condition on the lower level problem (condition 4.), it is clear that $q_{L}^{1}$ and $q_{L}^{2}$ cannot be zero on $\left[t_{a}, 6\right]$, otherwise, the maximum would be attained at $u_{1}^{*}=u_{2}^{*}=0$, contradicting the expressions of the optimal controls in (8), and 9) on $[0.254,6]$. This confirms the nontriviality of multipliers. Hence, the necessary optimality conditions postulated in Theorem 3.1 are satisfied at $\left(\bar{v}_{1}^{*}, \bar{v}_{2}^{*}, u_{1}^{*}, u_{2}^{*}\right)$.

\section{CONCLUSION}

In this work, we investigated the structured crowd motion model formulated as a bilevel control sweeping problem. We established the necessary optimality conditions when several participants are involved. To better understand those conditions, we illustrate an example with only two participants, for which we find the optimal solution explicitly. We showed that the necessary optimality conditions are satisfied for the computed optimal solution.

For the future research, we are interested in investigating a setting of problem where a sweeping process is incorporated in the dynamics of the upper level as well. This occurs for instance if we consider $m$ groups of crowd motion models, where each group $i \in\{1, \ldots, m\}$ is following its own agent, and structured into $N^{i}$ subgroups. At the upper level, while each agent tends to reach its target with the minimal possible time, the sweeping process arises so that each agent guarantees the non-overlapping of his/her own group with the others. At the lower level, the subgroups will follow their own agents with the minimum effort while keeping a safe distance with the agent and with the other subgroups of the same group. This translates into a sweeping process in the corresponding dynamics at the lower level too.

\section{ACKNOWLEDGMENT}

Tan H. Cao acknowledges the support of the National Research Foundation of Korea grant funded by the Korea Government (MIST) NRF-2020R1F1A1A01071015.

N.T. Khalil and F.L. Pereira acknowledge the support of FCT R\&D Unit SYSTEC - POCI-01-0145-FEDER-006933, and MAGIC - POCI-01-0145- FEDER-032485 - funded by ERDF - COMPETE2020 - FCT/MEC - PT2020, STRIDE NORTE-01-0145-FEDER-000033.

Boris S. Mordukhovich acknowledges the sup-port of the USA National Science Foundation under grants DMS1007132 and DMS-1512846, by the USA Air Force Office of Scientific Research grant \#15RT0462, and by the Australian Research Council under Discovery Project DP-190100555.

Dao Nguyen acknowledges the support of the USA National Science Foundation under grant DMS-1512846, and by the USA Air Force Office of Scientific Research grant \#15RT0462.

\section{REFERENCES}

[1] Khalil, N.T. and Pereira, F.L. A Framework for the Control of Bilevel Sweeping Processes, Proceedings of the 58th IEEE Conference on Decision and Control, CDC (2019).

[2] Khalil, N.T. and Pereira, F.L. A Maximum Principle for a time-optimal bi-level sweeping control problem, Published in IFAC (2020).

[3] Cao, T. H. and Mordukhovich, B. S. (2019). Applications of optimal control of a nonconvex sweeping process to optimization of the planar crowd motion model. Discrete \& Continuous Dynamical Systems-B, 24(8), 4191.

[4] Mordukhovich, B. S. Variational analysis and generalized differentiation I: Basic theory (Vol. 330). Springer Science \& Business Media 2006.

[5] Clarke, F.H. Optimization and Nonsmooth Analysis (Vol. 5). SIAM, 1990.

[6] Vinter, R. Optimal control. Springer Science \& Business Media 2010.

[7] Ye, J. J. Optimal strategies for bilevel dynamic problems. SIAM journal on control and optimization, 35(2), 512-531 (1997).

[8] Benita, F., Dempe, S., Mehlitz, P. Bilevel optimal control problems with pure state constraints and finite-dimensional lower level. SIAM Journal on Optimization, 26(1), 564-588 (2016).

[9] Dubovitskii, A.Ya., Milyutin, A.A. Extremum problems in the presence of restrictions, USSR Computational Mathematics and Mathematical Physics 5(3), 1-80 (1965).

[10] Gamkrelidze, R.V. Time-optimal process with bounded phase coordinates. Dokl. Akad. Nauk SSSR, 125(3), 475-478 (1959).

[11] Gamkrelidze, R.V. Optimal control processes under bounded phase coordinates. Izv. Akad. Nauk SSSR, Ser. Mat., 24(3), 315-356 (1960).

[12] Arutyunov, A.V., Karamzin, D.Yu., Lobo Pereira, F. The Maximum Principle for Optimal Control Problems with State Constraints by R.V. Gamkrelidze: Revisited. J. Optimization Theory and Applications, 149, 474-493 (2011).

[13] Arutyunov, A.V., Karamzin, D.Yu., Lobo Pereira, F. Investigation of controllability and regularity conditions for state constrained problems. IFAC-PapersOnLine, 50(1), 6295-6302 (2017).

[14] Karamzin, D.Yu., Lobo Pereira, F. On a Few Questions Regarding the Study of State-Constrained Problems in Optimal Control. Journal of Optimization Theory and Applications, 180(1), 235-255 (2019). 